\title{
Punctal Stenosis a Rare Complication of Dupilumab Therapy for Atopic Dermatitis: A New Case Report
}

\author{
Waqas S. Abdulwahhab ${ }^{1 *}$, Fatima Ibrahim Alamiri², Alaa S. Mehair ${ }^{1}$, Fatma Abdulghaffar Qaderi ${ }^{1}$ \\ ${ }^{1}$ Department of Dermatology and Venereology, Al Qassimi Hospital, Sharjah, United Arab Emirates \\ ${ }^{2}$ Department of Ophthalmology, Al Qassimi Hospital, Sharjah, United Arab Emirates \\ Email: *wqs_saad@yahoo.com,drfatimauae@gmail.com, alaa.alazawe@yahoo.com, fatma-959@hotmail.com
}

How to cite this paper: Abdulwahhab, W.S., Alamiri, F.I., Mehair, A.S. and Qaderi, F.A. (2021) Punctal Stenosis a Rare Complication of Dupilumab Therapy for Atopic Dermatitis: A New Case Report. Journal of Cosmetics, Dermatological Sciences and Applications, 11, 96-100.

https://doi.org/10.4236/jcdsa.2021.112010

Received: April 9, 2021

Accepted: June 1, 2021

Published: June 4, 2021

Copyright $\odot 2021$ by author(s) and Scientific Research Publishing Inc. This work is licensed under the Creative Commons Attribution International License (CC BY 4.0).

http://creativecommons.org/licenses/by/4.0/

\begin{abstract}
Background: Dupilumab is the biological drug approved for the treatment of moderate to severe atopic dermatitis (AD) and has demonstrated impressive clinical effect and quality of life-enhancing capacity in clinical trials. The most commonly observed adverse effects of dupilumab therapy included upper respiratory tract infection, headache, nasopharyngitis, injection-site reaction, herpes viral infection, and conjunctivitis. Lacrimal duct obstruction or punctal stenosis is rarely reported side effect of dupilumab therapy and not fully documented. Aim: To document a new case presentation of a young female with a history of $\mathrm{AD}$ without previous significant ocular manifestations who developed right eye punctal stenosis while she was on Dupilumab therapy for a one-year duration. Case Report: A 19-year-old female with a long-standing history of $\mathrm{AD}$ and on dupilumab therapy for one year duration who developed severe punctal stenosis and continued tearing from her right eye in the last two months not responded to conservative ophthalmological medications but completely improved on discontinuation dupilumab injection over 6 months followed-up. Conclusions: Conjunctivitis is a well-known adverse effect of Dupilumab injection of a patient with AD. However, persistent conjunctivitis and tearing from the eye not improving on ophthalmology treatment might rule out punctal stenosis and discontinuation of dupilumab should be considered.
\end{abstract}

\section{Keywords}

Atopic Dermatitis, Conjunctivitis, Dupilumab, Punctual Stenosis

\section{Introduction}

$\mathrm{AD}$ is a common chronic inflammatory skin disease characterized by a T-cell 
(Th2)-mediated immune response and epidermal dysfunction [1]. Dupilumab is a recent biological treatment of moderate to severe $\mathrm{AD}$. It is a human monoclonal antibody that blocks the shared interleukin (IL)- 4 receptor $\alpha$ subunit, thereby inhibiting the signaling of IL-4 and IL-13, type 2 (Th2) cytokines [2]. It has demonstrated impressive clinical effect and quality of life-enhancing capacity in clinical trials [3]. The most commonly observed adverse effects of dupilumab therapy included upper respiratory tract infection, headache, nasopharyngitis, injection-site reaction, herpes viral infection, and conjunctivitis [4]. However, reports of additional ocular side effects continue to emerge, and recommendations for the management of these side effects remain undefined [5] [6]. The association between punctal stenosis and Dupilumab therapy is rarely reported [5].

In the present case report, we are describing a 19-year-old female with a longstanding history of $\mathrm{AD}$ and on dupilumab therapy for one-year duration who developed severe punctal stenosis and continued tearing from the right eye in the last two months that didn't respond to conservative ophthalmological medications but completely improved on discontinuation dupilumab injection over 6 months followed-up. The consent form was taken from the patient about the publication of her condition.

\section{Case Report}

A 19-year-old female patient with a long-lasting history of $\mathrm{AD}$ and on Dupilumab therapy $300 \mathrm{mg}$ subcutaneously every two weeks for the one-year duration, presented to our clinic with the persistent red right eye and continuing tearing for the last two months. According to history, at initial first few months of treatment with dupilumab, presented with a bilateral mild conjunctival injection which improved on topical eye lubricants. On examination, she had severe irritation, redness of the conjunctiva, blurred vision, and continuing tearing from her right eye (Figure 1). The patient Referred to the ophthalmology clinic for further assessment. During ophthalmological examination; palpation of the right lacrimal sac revealed distension and expression of fluid from the puncta, thus diagnosing obstruction and blockage proximal to the lacrimal sac. Fluorescein dye disappearance test (FDDT) indicates impaired drainage in the right eye. Thus the diagnosis of right punctal stenosis (lacrimal duct obstruction) was confirmed. Carbomer 0.2\% eye gel, Cyclosporine 0.05\% (0.4 mL) eye drop, Naphazoline-Pheniramine eye drops, and Hyaluronate Sodium 0.2\% Minim drops were prescribed, but there was no improvement and her condition became worse. After discussion with the ophthalmologist, the decision for discontinuation dupilumab injection and planning for surgical intervention with probing, punctoplasty, and silicone intubation was recommended. The patient followed up at the clinic after one month with continued on just topical eye lubricants and stopped dupilumab injection. On examination, gradual improvement in signs and symptoms of punctal stenosis appeared where decreased conjunctival redness, tearing, and irritation with normal eye vision. Later six months followed-up there 
was a completely normal right eye, no redness or continuing tearing, and on ophthalmology examination normal lacrimal duct opening and resolved any signs for stenosis or obstruction (Figure 2). Regarding $\mathrm{AD}$, the patient was improved previously while she was on dupilumab with continued improvement despite stopped therapy. It is recommended to the patient to continue on just moisturization and following-up at the clinic in case of $\mathrm{AD}$ relapse.

\section{Discussion}

Dupilumab is a human monoclonal antibody that blocks the interleukin-4 receptor a subunit thereby inhibiting interleukin-4 (IL-4) and interleukin-13 (IL-13) signaling [2] [7] [8]. The association between conjunctivitis and dupilumab has been well-reported [9] [10]. In AD trials, the incidence of conjunctivitis ranged from $4.7 \%$ to $22.1 \%$ in dupilumab-treated patients, most cases were generally mild to moderate, and resolved without the need for discontinuation of dupilumab [9]. The mechanisms for dupilumab-associated eye complications remain unclear. Several hypotheses have been proposed to explain incident conjunctivitis during dupilumab treatment, including eosinophilia, increased OX40 ligand activity, and a dysregulated immune response in conjunctival-associated

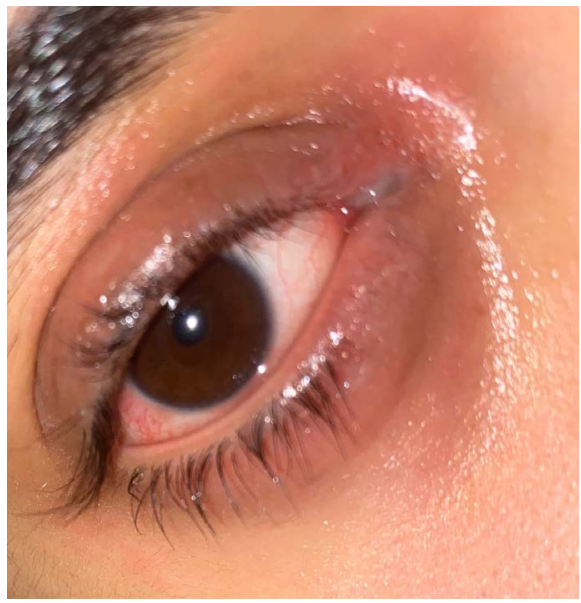

Figure 1. Showed upper and lower eyelids inflammation with edema, conjunctival injection, and signs of lacrimal duct obstruction with continuous tearing from the right eye of a 19-year-old female patient with a history of AD.

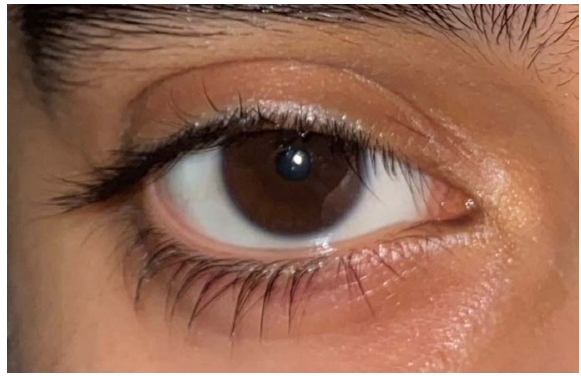

Figure 2. Showed normal-appearing right eye six months after discontinued dupilumab injection in a 19-year-old female patient with a history of AD. 
lymphoid tissue [9]. The association between punctal stenosis and Dupilumab therapy is rarely reported [5] [11]. The pathogenesis of punctal stenosis may involve chronic inflammation from conjunctivitis leading to cicatrix and gradual fibrotic change of external puncta with eventual stenosis [12].

Another hypothesis may be that dupilumab is secreted in tears and on direct contact, induces inflammation and closure of the lacrimal drainage apparatus resembling the mechanism postulated in docetaxel-associated canalicular stenosis [13].

The reason for lacrimal duct obstruction in this condition might be due to inflammation and hypertrophy of the underlying endothelium leads to stenosis and later fibrosis which if diagnosed early can be treated conservatively without surgical intervention.

Additional more significant ocular complications that have emerged in association with Dupilumab therapy are cicatricial conjunctiva and ectropion [5] [6].

In all reported cases conjunctivitis and punctal stenosis were bilateral [5] [11], while in our case it was unilateral involving the right eye.

In our patient, complete resolution of conjunctivitis and punctal stenosis occurs within six months after discontinuation of dupilumab as reported in the literature [5].

For dupilumab-induced conjunctivitis, initial management has proposed ophthalmology evaluation, ophthalmic preparations of antihistamines, corticosteroids, or immunosuppressive agents, and dupilumab taper or discontinuation [6].

In conclusion, conjunctivitis is a well-known adverse effect of Dupilumab injection of a patient with AD. However, persistent conjunctivitis and tearing from the eye not improving on ophthalmology treatment might rule out punctal stenosis and discontinuation of dupilumab should be considered. Additional studies are required to document the possibility of developing punctal stenosis in patients using dupilumab for cases other than $\mathrm{AD}$, like nasal polyps and asthma.

\section{Disclosure}

This study is an independent study and not funded by any of the drug companies.

\section{Conflicts of Interest}

The authors declare no conflicts of interest regarding the publication of this paper.

\section{References}

[1] Weidinger, S. and Novak, N. (2016) Atopic Dermatitis. Lancet, 387, 1109-1122. https://doi.org/10.1016/S0140-6736(15)00149-X

[2] US-FDA (2019) FDA Labeling Information-DUPIXENT. FDA website. https://www.accessdata.fda.gov/drugsatfda_docs/label/2018/761055s007lbl.pdf

[3] Ivert, L.U., Wahlgren, C.F., Ivert, L., Lundqvist, M. and Bradley, M. (2019) Eye 
Complications during Dupilumab Treatment for Severe Atopic Dermatitis. Acta Dermato-Venereologica, 99, 375-378. https://doi.org/10.2340/00015555-3121

[4] Paller, A.S., Bansal, A., Simpson, E.L., Boguniewicz, M., Blauvelt, A., Siegfried, E.C., Guttman-Yassky, E., Hultsch, T., Chen, Z., Mina-Osorio, P., Lu, Y., Rossi, A.B., He, X., Kamal, M., Graham, N.M.H., Pirozzi, G., Ruddy, M., Eckert, L. and Gadkari, A. (2020) Clinically Meaningful Responses to Dupilumab in Adolescents with Uncontrolled Moderate-to-Severe Atopic Dermatitis: Post-hoc Analyses from a Randomized Clinical Trial. American Journal of Clinical Dermatology, 21, 119-131. https://doi.org/10.1007/s40257-019-00478-y

[5] Barnes, A.C., Blandford, A.D. and Perry, J.D. (2017) Cicatricial ectropion in a Patient Treated with Dupilumab. American Journal of Ophthalmology Case Reports, 7, 120-122. https://doi.org/10.1016/j.ajoc.2017.06.017

[6] Levine, R.M., Tattersall, I.W., Gaudio, P.A., et al. (2018) Cicatrizing Blepharoconjunctivitis Occurring during Dupilumab Treatment and a Proposed Algorithm for Its Management. JAMA Dermatology, 154, 1485-1486.

https://doi.org/10.1001/jamadermatol.2018.3427

[7] Simpson, E.L., Bieber, T., Guttman-Yassky, E., et al. (2016) Two Phases 3 Trials of Dupilumab Versus Placebo in Atopic Dermatitis. The New England Journal of Medicine, 375, 2335-2348. https://doi.org/10.1056/NEJMoa1610020

[8] de Bruin-Weller, M., Thaçi, D., Smith, C.H., et al. (2018) Dupilumab with Concomitant Topical Corticosteroids in Adults with Atopic Dermatitis with an Inadequate response or Intolerance to Cyclosporin A or When This Treatment Is Medically Inadvisable: A Placebo-Controlled, Randomized Phase III Clinical Trial (LIBERTY AD CAF_E). British Journal of Dermatology, 178, 1083-1101. https://doi.org/10.1111/bjd.16156

[9] Akinlade, B., Guttman-Yassky, E., de Bruin-Weller, M., et al. (2019) Conjunctivitis in Dupilumab Clinical Trials. British Journal of Dermatology, 181, 459-473. https://doi.org/10.1111/bjd.17869

[10] Treister, A.D., Kraff-Cooper, C. and Lio, P.A. (2018) Risk Factors for Dupilumab-Associated Conjunctivitis in Patients with Atopic Dermatitis. JAMA Dermatology, 154, 1208-1211. https://doi.org/10.1001/jamadermatol.2018.2690

[11] Lee, D.H., Cohen, L.M., Yoon, M.K. and Tao, J.P. (2020) Punctal Stenosis Associated with Dupilumab Therapy for Atopic Dermatitis. Journal of Dermatological Treatment. https://doi.org/10.1080/09546634.2019.1711010

[12] Soiberman, U., Kakizaki, H., Selva, D., et al. (2012) Punctal Stenosis: Definition, Diagnosis, and Treatment. Clinical Ophthalmology, 6, 1011-1018. https://doi.org/10.2147/OPTH.S31904

[13] Esmaeli, B., Ahmadi, M., Rivera, E., et al. (2002) Docetaxel Secretion in Tears: Association with Lacrimal Drainage Obstruction. Archives of Ophthalmology, 120, 1180-1182. https://doi.org/10.1001/archopht.120.9.1180 